\title{
Review
}

\section{Identification of new genetic risk factors for prostate cancer}

Michelle Guy ${ }^{1}$, Zsofia Kote-Jarai ${ }^{1}$, Graham G. Giles ${ }^{2,3}$, Ali Amin Al Olama ${ }^{4}$, Sarah K. Jugurnauth ${ }^{1}$, Shani Mulholland ${ }^{1}$, Daniel A. Leongamornlert ${ }^{1}$, Stephen M. Edwards ${ }^{1}$, Jonathan Morrison ${ }^{4}$, Helen I. Field ${ }^{5}$, Melissa C. Southey ${ }^{6}$, Gianluca Severi ${ }^{2,3}$, Jenny L. Donovan ${ }^{7}$, Freddie C. Hamdy ${ }^{8}$, David P. Dearnaley ${ }^{1,9}$, Kenneth R. Muir ${ }^{10}$, Charmaine Smith ${ }^{2}$, Melisa Bagnato ${ }^{2}$, Audrey T. Ardern-Jones ${ }^{9}$, Amanda L. Hall ${ }^{1,9}$, Lynne T. O’Brien ${ }^{1}$, Beatrice N. Gehr-Swain ${ }^{1,9}$, Rosemary A. Wilkinson ${ }^{1}$, Angela Cox ${ }^{11}$, Sarah Lewis ${ }^{7}$, Paul M. Brown ${ }^{12}$, Sameer G. Jhavar ${ }^{1}$, Malgorzata Tymrakiewicz ${ }^{1}$, Artitaya Lophatananon ${ }^{10}$, Sarah L. Bryant ${ }^{1}$, The UK Genetic Prostate Cancer Study Collaborators ${ }^{13}$, British Association of Urological Surgeons' Section of Oncology and The UK ProtecT Study Collaborators ${ }^{13}$, Alan Horwich ${ }^{1,9}$, Robert A. Huddart ${ }^{1,9}$, Vincent S. Khoo ${ }^{1,9}$, Christopher C. Parker, ${ }^{1,9}$, Christopher J. Woodhouse ${ }^{9}$, Alan Thompson ${ }^{9}$, Tim Christmas ${ }^{9}$, Chris Ogden ${ }^{9}$, Cyril Fisher', Charles Jameson $^{9}$, Colin S. Cooper ${ }^{1}$, Dallas R. English ${ }^{3}$, John L. Hopper ${ }^{3}$, David E. Neal ${ }^{12,14}$, Douglas F. Easton ${ }^{4}$, Rosalind A. Eeles ${ }^{1,9}$

${ }^{I}$ Section of Cancer Genetics, The Institute of Cancer Research, Sutton, Surrey SM2 5NG, UK; ${ }^{2}$ Cancer Epidemiology Centre, The Cancer Council Victoria, Carlton VIC 3053, Australia; ${ }^{3}$ Centre for Molecular, Environmental, Genetic and Analytic Epidemiology, The University of Melbourne, Carlton VIC 3053, Australia; ${ }^{4}$ Cancer Research UK Genetic Epidemiology Unit, University of Cambridge, Strangeways Laboratory, Worts Causeway, Cambridge CB1 8RN, UK; ${ }^{5}$ Department of Oncology, University of Cambridge, Strangeways Laboratory, Worts Causeway, Cambridge CB1 8RN, UK; ${ }^{6}$ Genetic Epidemiology Laboratory, Department of Pathology, The University of Melbourne, Parkville VIC 3052, Australia; ${ }^{7}$ Department of Social Medicine, University of Bristol, Bristol BS8 2PR, UK; ${ }^{8}$ Academic Urology Unit, University of Sheffield, Sheffield S10 2JF, UK; ${ }^{9}$ The Royal Marsden NHS Foundation Trust, Sutton, Surrey SM2 5PT and London SW3 6JJ, UK; ${ }^{10}$ University of Nottingham Medical School, Queens Medical Centre, Nottingham NG7 2UH, UK; ${ }^{11}$ Institute for Cancer Studies, University of Sheffield, Sheffield S10 2RX, UK; ${ }^{12}$ Surgical Oncology (Uro-Oncology S4), Departments of Oncology and Surgery, University of Cambridge, Addenbrooke's Hospital, Cambridge CB2 2QQ, UK; ${ }^{13} \mathrm{~A}$ full list of authors is provided in the Supplementary Note online; ${ }^{14}$ Cancer Research UK Cambridge Research Institute, Li Ka Shing Centre, Cambridge CB2 ORE, UK.

\begin{abstract}
There is evidence that a substantial part of genetic predisposition to prostate cancer ( $\mathrm{PCa}$ ) may be due to lower penetrance genes which are found by genome-wide association studies. We have recently conducted such a study and seven new regions of the genome linked to PCa risk have been identified. Three of these loci contain candidate susceptibility genes: $M S M B, L M T K 2$ and $K L K 2 / 3$. The $M S M B$ and $K L K 2 / 3$ genes may be useful for PCa screening, and the LMTK2 gene might provide a potential therapeutic target. Together with results from other groups, there are now 23 germline genetic variants which have been reported. These results have the potential to be developed into a genetic test. However, we consider that marketing of tests to the public is premature, as PCa risk can not be evaluated fully at this stage and the appropriate screening protocols need to be developed. Follow-up validation studies, as well as studies to explore the psychological implications of genetic profile testing, will be vital prior to roll out into healthcare.
\end{abstract}

Asian Journal of Andrology (2009) 11: 49-55. doi: 10.1038/aja.2008.18; published online 1 December 2008.

Keywords: prostate cancer, genetics, susceptibility loci, SNPs, relative risks

Correspondence to: Dr Michelle Guy, Translational Cancer Genetics Team, The Institute of Cancer Research, 15 Cotswold Road, Sutton, Surrey SM2 5NG, UK.

Tel: +44-20-8661-3507_ E-mail: Michelle.Guy@icr.ac.uk

Received: 30 September 2008 Accepted: 2 October 2008

Published online: 1 December 2008

\section{Genome-wide association studies}

Genome-wide association studies (GWAS) have recently emerged as a powerful new approach to identify common disease alleles. These studies have been made 
possible following the completion of the International HapMap Project in 2005 [1] which provided researchers with a computerized database that contains a map of human genetic variation, or tagSNPs. There are millions of single-nucleotide polymorphisms (SNPs) in the human genome; these are classed as a variation in a single nucleotide (A, T, C or G) in the DNA sequence between members of a species. The variation must be present in at least $1 \%$ of a population for it to be considered an SNP. It is not currently feasible to genotype every single SNP from a person's genome, but tagSNPs represent blocks within the genome with high linkage disequilibrium (LD), and can be used to rapidly scan a person's DNA to find genetic variations associated with particular diseases. GWAS have also recently become accessible to more research groups due to the development of high-throughput SNP arrays and the decreasing costs of this rapidly-advancing technology.

The first phases of a large UK GWAS on PCa have been completed this year by scientists at the Institute of Cancer Research and University of Cambridge in collaboration with researchers in Australia and the UK. This research has resulted in the identification of seven new sites in the human genome that are linked to a risk of developing $\mathrm{PCa}$, and the findings have been published in Nature Genetics [2] alongside two other studies carried out in the United States [3] and Iceland [4].

\section{Prostate cancer risk and genetics}

$\mathrm{PCa}$ is now the commonest cancer in men in the Western world (Cancer Research UK Factsheets, 2008). However, its etiology remains poorly understood and no definite lifestyle risk factors have been identified. PCa shows consistent evidence of familial aggregation and it is known that the relative risk (RR) of PCa is about two-fold in first-degree relatives of affected men, and that this is higher when the diagnosis is of early-onset (reviewed in [5]). In contrast to some other cancer types, little is known about which genes could be involved in PCa susceptibility. In a very small number of cases, $\mathrm{PCa}$ is associated with germline alterations in the $B R C A 2$ gene [6] and other genes in the DNA repair pathway, however these mutations explain less than $10 \%$ of the familial relative risk of PCa. Linkage studies based on multiple case families have not identified reproducible susceptibility loci [7], suggesting that $\mathrm{PCa}$ is genetically complex and that $\mathrm{PCa}$ predisposition may be mediated through multiple common low-penetrance alleles. A more powerful approach for identifying lower penetrance loci is through case-control GWAS, and previous GWAS in PCa have identified common alleles on $8 \mathrm{q}$ and on $17 \mathrm{q}$ to be associated with PCa risk [8-18]. Chromosome 8q24 has been found to contain multiple SNPs associated with risk of PCa [19] and other cancers, including colon, breast and ovarian [20], but interestingly appears to have no genes in the region of interest. Further studies are underway by many research groups to look for genes in this region, or work out a mechanism by which these genotypes might result in increasing cancer risk.

Dr Rosalind Eeles and her team at the Institute of Cancer Research have been studying the genetic causes of PCa since 1993 when the Familial Prostate Cancer study, now known as the UK Genetic Prostate Cancer Study (UKGPCS), was established. The UKGPCS looks at three different sets of patients; a subset with a family history of $\mathrm{PCa}$, a subset with early-onset PCa (diagnosed aged $\leq 60$ years, with or without a family history) and a series of $\mathrm{PCa}$ patients from the Royal Marsden NHS Foundation Trust (diagnosed at any age, with or without a family history). Using DNA samples from these patients, it is hoped to find susceptibility genes with different levels of penetrance.

\section{Collection of case and control samples}

In GWAS studies, genotype frequencies are compared between cases and controls at large numbers of tagSNPs in order to look for a variation that is associated with disease. We conducted a two-stage PCa GWAS using samples amalgamated from four collaborative studies. UK cases were provided from the UKGPCS run at the Institute of Cancer Research by Dr Rosalind Eeles, with substantial support from Cancer Research UK. UK controls were from both the Prostate Cancer Research Foundation component of the UKGPCS (coordinated by Professor Kenneth Muir at the University of Nottingham, UK) and from the ProtecT study, a national study of communitybased PSA testing and a randomized trial of subsequent PCa treatment that is led by Professor David Neal at the University of Cambridge (UK), and funded by Cancer Research UK and the Department of Health of England (UK). Further data collection was funded by Prostate UK. Australian samples were provided from three studies: the Early-Onset Prostate Cancer Study (EOPCFS), the Risk Factors for Prostate Cancer Study (RFPCS), and the Melbourne Collaborative Study (MCCS). These samples were collected by Professor John Hopper at the University of Melbourne (Australia) and Professor Graham Giles at the Cancer Council Victoria (Australia) with local Australian funding. Case and control samples have to be matched ethnically as closely as possible to avoid adding any extra bias to the experiment. Therefore we chose only Caucasian samples of European ancestry and excluded known non-whites so that the ethnic background of samples was homogeneous. In addition, we broadly matched cases and controls geographically in the UK for 
stage 1 to reduce as far as possible any bias generated by population substructure.

\section{Analysis of samples}

In the first stage of the UK GWAS, we genotyped 2000 case and 2000 control samples and received usable data on 1854 cases and 1894 controls. For this first stage we specifically selected cases that were diagnosed through clinical symptoms rather than through routine screening by PSA, in order to maximize the proportion of cases that cause morbidity and mortality. The case series was further 'enriched' by including men diagnosed at early onset (age $\leq 60$ years) or with a family history of PCa, as such individuals are thought to be more likely to carry susceptibility alleles, and including them in the GWAS would thereby increase statistical power. Controls were men aged $\geq 50$ years and who had a baseline PSA $<0.5 \mathrm{ng} \mathrm{mL}^{-1}$, since men with low PSA concentrations are known to be at a low risk for the subsequent development of clinically significant $\mathrm{PCa}$.

For each sample, data were collected on 541129 tagSNPs using the Illumina Infinium platform, and analysis of this data was carried out by Professor Douglas Easton and Ali Amin from the University of Cambridge (UK). Our stage 1 results showed that a total of 53 tagSNPs were significant at the $P<10^{-6}$ level compared with 0.5 expected by chance, therefore pulling out more hits that we had anticipated at this stage. Of these, 20 tagSNPs were on chromosome 8q, and 6 were on chromosome $17 \mathrm{q}$, both of which had previously been reported by other research groups $[8,12]$. However, the other 27 tagSNPs were found to be in 8 novel genetic regions and after further analyses, 11 of the tagSNPs were independently significant (not in LD with other SNPs).

To confirm the associations from stage 1, our laboratory team at the Institute of Cancer Research evaluated the 11 independently significant SNPs using the Taqman assay in DNA samples that had been collected for our second stage of the GWAS. We produced genotype data for a further $3268 \mathrm{PCa}$ cases and 3366 controls. Using a stringent $P$ value for genome-wide significance accepted to be a cutoff of $P<10^{-7}$, the associations were confirmed in 8 of the 11 SNPs, and these were located in seven loci (two were on chromosome 10). These seven newly identified susceptibility regions are on human chromosomes 3, 6, 7, 10, 11, 19 and $\mathrm{X}$. Figure 1 shows a summary of these results.

\section{Identification of candidate genes within loci}

The seven novel genetic loci found in the UK GWAS contain several plausible candidate genes, which could functionally contribute to cancer development and progression. The top association was found on chromosome

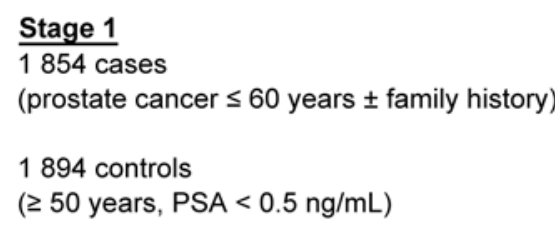

Stage 2

3268 cases 3366 controls (not selected for PSA) Typed for further SNPs
550000 SNPs

541129 SNPs passed quality control
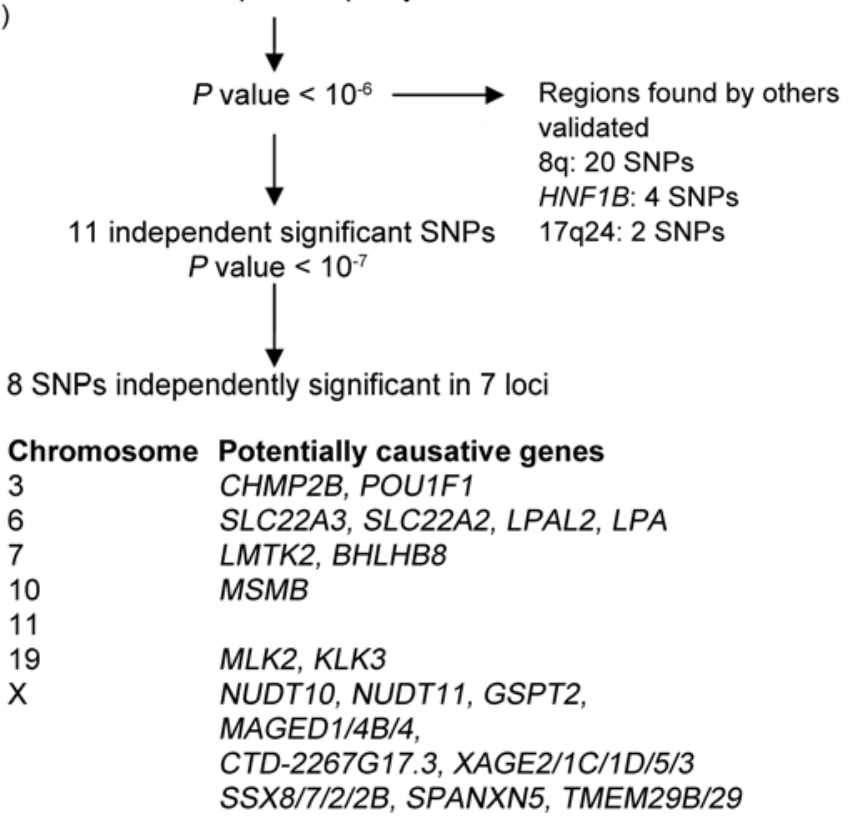

Figure 1. Summary of the UK prostate genome-wide association studies (GWAS) study and results. 
10 , just 2 bp upstream of the transcription start site of the microseminoprotein B (MSMB) gene. MSMB encodes PSP94, a member of the immunoglobulin-binding factor family synthesized by epithelial cells of the prostate and secreted into seminal plasma. Loss of expression of PSP94 is associated with recurrence after radical prostatectomy, suggesting that this SNP may be causally related to disease risk [21]. Since PSP94 can be measured in the blood this may provide a future screening target.

The region identified on chromosome 7 harbors the gene LMTK2 (also known as BREK; Brain-Enriched Kinase) which codes for a signaling protein [22] and therefore may be a new target for drug treatments.

The chromosome 19 hit contains the kallikrein genes $K L K 2$ and $K L K 3$. These code for the proteins hK2 (human glandular kallikrein) and prostate-specific antigen (PSA) respectively, which are serine proteases. PSA is used as serum marker in $\mathrm{PCa}$ screening and disease monitoring and there is evidence that hK2 may also be useful for screening and prognosis [23]. Twenty-four SNPs in the $K L K 3$ (PSA) gene have subsequently been evaluated in men from five studies and no association was reported with $\mathrm{PCa}$ risk [24]. In our stage 1, the controls were selected for low PSA levels, however, the stage 2 controls were not selected for a low PSA and yet we still saw an association. A subsequent study from 13 groups worldwide where the controls were not selected for a low PSA level, still showed an association of the chromosome 19 SNP (between $K L K 2$ and $K L K 3$ ) with PCa risk, so we consider this to be a real effect [25].

The chromosome 6 association is in intron 5 of $S L C 22 A 3$, one of the organic cation transporter (OCT) genes, which are critical for elimination of some drugs and environmental toxins [26]. Many genes are near the SNP of interest on the $\mathrm{X}$ chromosome and are listed in Figure 1. The NUDT10 and NUDT11 genes encode enzymes that determine the rate of phosphorylation in DNA repair, stress responses and apoptosis [27]. The SNP found in chromosome 3 lies in a gene-poor region and the chromosome 11 hit is in a gene desert, making it difficult to associate these with obvious genes at this stage in the research.

The results so far confirm that PCa is genetically complex and have given us clues as to which regions of the genome to explore further. The fact that the SNPs which have been identified are in a variety of areas within the genome, for instance introns and gene deserts, and none are in coding regions of DNA, suggests that diverse pathways are likely to be involved. Resequencing and fine-mapping of all of the seven regions, as well as further genotyping and functional analyses, will be required to identify the causal variants.

6 Other prostate GWAS studies: how do our results compare?

Two other groups of researchers also published their PCa GWAS findings in the same issue of Nature Genetics as our team. These were a group called CGEMS in the USA [3] and the deCODE group in Iceland [4]. Both groups also confirmed the previously reported associations at chromosomes $8 \mathrm{q}$ and $17 \mathrm{q}$ in their sample sets, verifying these as strong hits in a global population. The American group also found the same hits as we did on chromosomes 10 and 11, but found novel tagSNP associations on chromosomes 7 and 10. The deCODE team identified the same region on the $\mathrm{X}$ chromosome as our study, and have found a novel region on chromosome $2 \mathrm{p} 15$ in their population.

One other GWAS has investigated aggressive PCas that were defined by having at least one of the following: stage T3/T4, N+, M+, Grade III, Gleason score $\geq 8$, or preoperative serum PSA of at least $50 \mathrm{ng} \mathrm{mL}^{-1}$ [28]. This group reported a different association on chromosome 9 located within the $D A B 2 I P$ gene, which encodes a novel Ras GTPase-activating protein and putative tumor suppressor. This phenotypic association remains to be validated by other research groups. In addition, this research group recently identified a second independent risk locus in chromosome $17 \mathrm{q} 12$ within the $H N F 1 B$ gene by fine-mapping of this region [29].

The results of the different GWAS studies are summarized in Figure 2, which shows the associations found by each study on each chromosome. The seven novel hits from the UK study are labeled in red, and the three purple arrows show the previously published associations that our results validated. With seven novel loci, our results represent the largest number of genetic risk factors found in one genome-wide cancer study to date. The fact that we have found more loci than the other groups, and also that we could confirm the previously reported loci at genomewide levels of significance after our stage 1 analysis is probably due to the size of the study, and perhaps also the enrichment of cases with early age at diagnosis or family history of PCa.

The GWAS groups are each already testing whether the hits from the other groups can be verified in their own datasets, and it is likely that many other loci will be detectable by further follow-up of this and other scans, together with combined analyses of multiple scans.

\section{Risk counseling for the future}

Our seven newly identified loci together explain approximately $6 \%$ of the familial risk of $\mathrm{PCa}$, with $M S M B$ being the most significant contributor $(\sim 2 \%$ of the familial risk, comparable to the two strongest $8 \mathrm{q}$ loci). Taken 


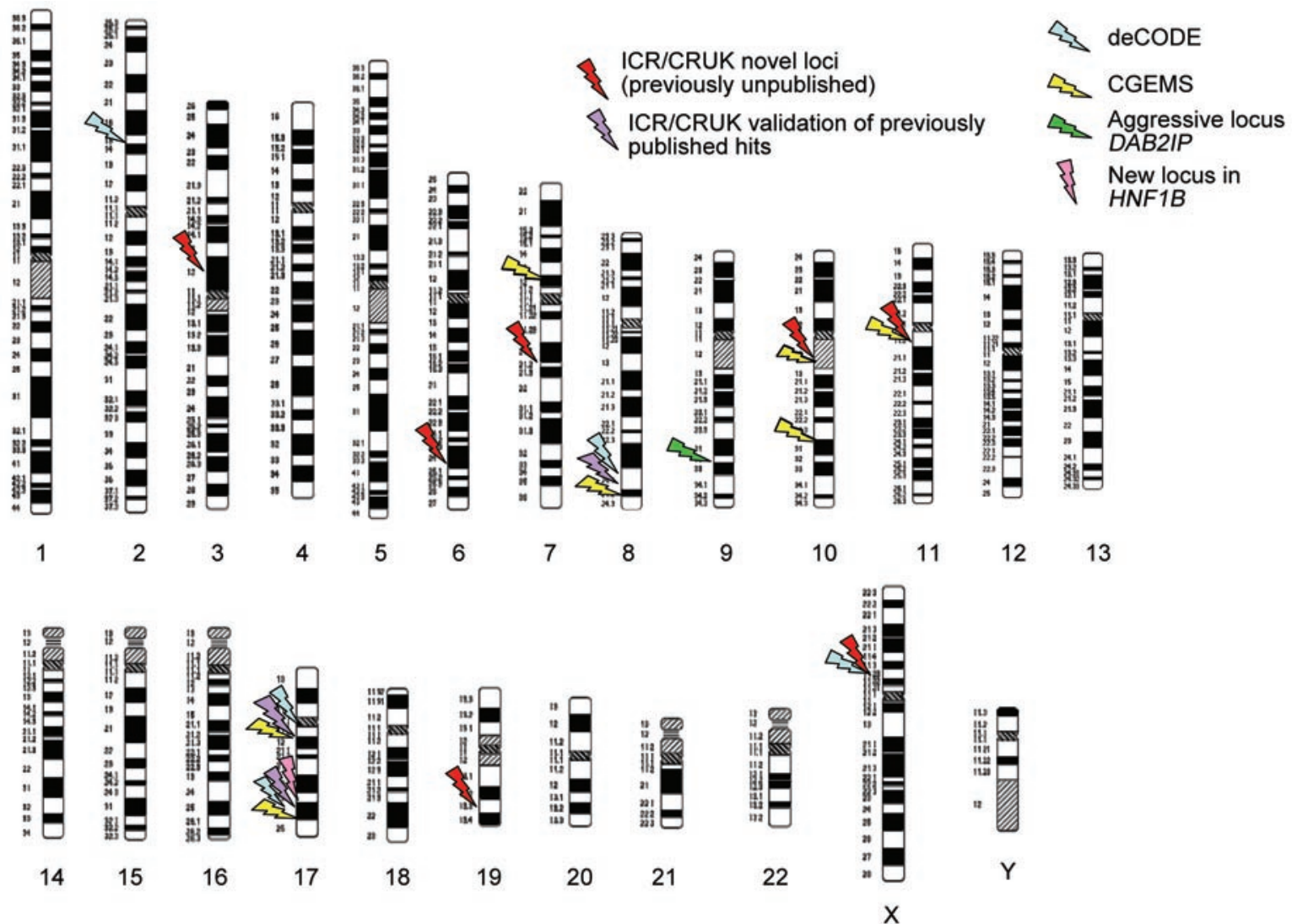

Figure 2. Summary of loci associated with prostate cancer (PCa) risk identified by our study and other genome-wide association studies (GWAS). (Figure drawn by Dr Kote-Jarai, The Institute of Cancer Research, Sutton, UK).

together, these and previously reported loci now explain approximately $15 \%$ of familial risk in PCa. As expected, the relative risks conferred by these loci individually are low, for instance, the homozygote odds ratio for the $M S M B$ hit was 1.61 -fold. It is possible, however, that the associations we have found with the tagSNPs will become stronger once the causal variant SNP has been pinpointed with fine-mapping. We also anticipate that the combined effect of these associations may be substantial, so that if a man has all seven risk variants in his DNA, he will have a much higher risk of developing PCa than a man with only two risk variants for example. As other new SNPs are identified, it may be possible in the future to define genotypes that are sufficiently predictive of risk to be of clinical use.

Eventually we would like to be able to use genotyping results to compile a genetic profile for a patient, so that we can accurately predict who has a high risk of developing PCa and therefore target screening to those men who are most likely to benefit. The results do have the potential to be developed into a genetic test, but at the moment the marketing of tests to the public is premature until the associations that have been found are further validated and better understood. In addition, further research needs to be carried out to identify how genetic profiling helps to tailor PCa screening regimes, for example we need to determine for a specific genotype, how often PSA testing would need to be done, and whether and when biopsy should be carried out. We also need to consider the acceptability of such tests and the psychological implications and these need careful study. We need to answer questions such as who would come forward for testing, how much counseling would they require, and what would they be advised to do if they were found to have a higher risk of developing $\mathrm{PCa}$ than the general population.

\section{Future work}

Although our stage 2 samples have been tested at the Institute of Cancer Research for the 11 independently significant SNPs that were flagged up at the end of stage 1 , this whole set has also been run at Illumina for another 47000 tagSNPs. This set of tagSNPs was selected by us as having the greatest differences between cases compared with controls in our stage 1 data. The results of the stage 
2 Illumina analyses will be ready shortly and may provide us with more novel hits to investigate further.

We are also validating the tagSNPs identified by the other GWAS studies in our own sample sets so that we can see whether there is variation perhaps between different populations, e.g. European and African ancestry. This question will also be addressed in our stage 3 GWAS where we intend to genotype 10000 cases and 10000 controls from different countries and ethnic backgrounds for the top hits from stages 1 and 2 to verify whether there are strong associations that affect all populations, or whether some populations have their own unique genotypic variations that confer an increased risk of $\mathrm{PCa}$. This may give us an explanation for the differences in $\mathrm{PCa}$ incidence and aggressiveness in black men compared to other ethnic groups.

The UKGPCS is continuing to collect early-onset and familial PCa cases to include in stage 3 of the UK GWAS. Currently over 130 hospitals throughout the UK have local approval to refer patients to the study, and all consultants who refer are added to the list of collaborators for publication purposes (over 300 collaborators). If you would like to refer patients to the study or find out more, please find our contact details and more information on our website at www.icr.ac.uk/ukgpes.

\section{Acknowledgment}

We should like to thank all the individuals (patients and controls) who took part in our study. Our work is supported by Cancer Research UK Grant C5047/A3354, and by the Biomedical Research Centre at the Institute of Cancer Research and Royal Marsden NHS Foundation Trust. DFE is a Principal Research Fellow of Cancer Research UK. JLH is an Australia Fellow of the NHMRC. We also thank the following for funding support: The Institute of Cancer Research and The Everyman Campaign, The Prostate Cancer Research Foundation, Prostate Research Campaign UK (now known as Prostate UK), The National Cancer Research Network UK, The National Cancer Research Institute (NCRI) UK, The National Health and Medical Research Council, Australia (209057, 251533, 450104), VicHealth, The Cancer Council Victoria, The Whitten Foundation, and Tattersall's. We acknowledge the contribution of all members of the UKGPCS, BAUS and ProtecT study research groups. The ProtecT study is ongoing and is funded by the Health Technology Assessment Programme (projects 96/20/06, 96/20/99). The ProtecT trial and its linked ProMPT and CAP (Comparison Arm for ProtecT) studies are supported by the Department of Health, England; Cancer Research UK grant C522/A8649, Medical Research Council of England grant G0500966, ID 75466 and The NCRI, UK. The epidemiological data for ProtecT were generated though funding from the Southwest National Health Service Research and Development. DNA extraction in Protec $\mathrm{T}$ was supported by the USA CDMRP award number W81XWH-04-1-280. The views and opinions expressed herein are those of the authors and do not necessarily reflect those of the Department of Health of England.

\section{References}

1 The International HapMap Consortium: A haplotype map of the human genome. Nature 2007; 437: 1299-320.

2 Eeles RA, Kote-Jarai Z, Giles GG, Olama AA, Guy M, et al. Multiple newly identified loci associated with prostate cancer susceptibility. Nat Genet 2008; 40: 316-21.

3 Thomas G, Jacobs KB, Yeager M, Kraft P, Wacholder S, et al. Multiple loci identified in a genome-wide association study of prostate cancer. Nat Genet 2008; 40: 310-5.

4 Gudmundsson J, Sulem P, Rafnar T, Bergthorsson JT, Manolescu $\mathrm{A}$, et al. Common sequence variants on $2 \mathrm{p} 15$ and Xp11.22 confer susceptibility to prostate cancer. Nat Genet 2008; 40: 281-3.

5 Edwards SM, Eeles RA. Unravelling the genetics of prostate cancer. Am J Med Genet C Semin Med Genet 2004; 129C: 65-73.

6 Edwards SM, Kote-Jarai Z, Meitz J, Hamoudi R, Hope Q, et al. Two percent of men with early-onset prostate cancer harbour germline mutations in the BRCA2 gene. Am J Hum Genet 2003; 72: 1-12.

7 Easton DF, Schaid DJ, Whittemore AS, Isaacs WJ, International Consortium for Prostate Cancer Genetics. Where are the prostate cancer genes? A summary of eight genome wide searches. Prostate 2003; 57: 261-9.

8 Amundadottir LT, Sulem P, Gudmundsson J, Helgason A, Baker A, et al. A common variant associated with prostate cancer in European and African populations. Nat Genet 2006; 38: 652-65.

9 Haiman CA, Patterson N, Freedman ML, Myers SR, Pike MC, et al. Multiple regions within $8 \mathrm{q} 24$ independently affect risk for prostate cancer. Nat Genet 2007a; 39: 638-44.

10 Haiman CA, Le Marchand L, Yamamato J, Stram DO, Sheng X, et al. A common genetic risk factor for colorectal and prostate cancer. Nat Genet 2007b; 39: 954-6.

11 Gudmundsson J, Sulem P, Manolescu A, Amundadottir LT, Gudbjartsson D, et al. Genome-wide association study identifies a second prostate cancer susceptibility variant at $8 \mathrm{q} 24$. Nat Genet 2007; 39: 631-7.

12 Gudmundsson J, Sulem P, Steinthorsdottir V, Bergthorsson JT, Thorleifsson G, et al. Two variants on chromosome 17 confer prostate cancer risk, and the one in TCF2 protects against type 2 diabetes. Nat Genet 2007; 39: 977-83.

13 Yeager M, Orr N, Hayes RB, Jacobs KB, Kraft P, et al. Genome-wide association study of prostate cancer identifies a second risk locus at 8q24. Nat Genet 2007; 39: 645-9.

14 Zheng SL, Sun J, Cheng Y, Li G, Hsu FC, et al. Association between two unlinked loci at $8 \mathrm{q} 24$ and prostate cancer risk among European Americans. J Natl Cancer Inst 2007; 99: 1525-33.

15 Schumacher FR, Feigelson HS, Cox DG, Haiman CA, Albanes D, et al. A common 8q24 variant in prostate and breast cancer from a large nested case control study. Cancer Res 2007; 67: 2951-6.

16 Wang L, McDonnell SK, Slusser JP, Hebbring SJ, Cunningham $\mathrm{JM}$, et al. Two common chromosome $8 \mathrm{q} 24$ variants are associated with increased risk for prostate cancer. Cancer Res 
2007; 67: 2944-55.

17 Suuriniemi M, Agalliu I, Schaid DJ, Johanneson B, McDonnell $\mathrm{SK}$, et al. Confirmation of a positive association between prostate cancer risk and a locus at chromosome 8q24. Cancer Epi Biomarkers Prev 2007; 16: 809-14.

18 Sun J, Lange EM, Isaacs SD, Liu W, Wiley KE, et al. Chromosome $8 \mathrm{q} 24$ risk variants in hereditary and non-hereditary prostate cancer patients. Prostate 2008; 68: 489-97.

19 Witte J. Multiple prostate cancer risk variants on 8q24. Nat Genet 2007; 39: 579-80.

20 Ghoussaini M, Song H, Koessler T, Amin Al Olama A, Kote-Jarai $\mathrm{Z}$, et al. Multiple loci with different cancer specificities within the 8q24 gene desert. J Natl Cancer Inst 2008; 100: 962-6.

21 Reeves JR, Dulude H, Panchal C, Daigneault L, Ramnani DM. Prognostic value of prostate secretory protein of 94 amino acids and its binding protein after radical prostatectomy. Clin Cancer Res 2006; 12: 6018-22.

22 Kawa S, Fujimoto J, Tezuka T, Nakazawa T, Yamamoto T. Involvement of BREK, a serine/threonine kinase enriched in brain, in NGF signalling. Genes Cells 2004; 9: 219-32.

23 Steuber T, Helo P, Lilja H. Circulating biomarkers for prostate cancer. World J Urol 2007; 25: 111-9.

24 Ahn J, Berndt SI, Wacholder S, Kraft P, Kibel AS, et al.
Variation in KLK genes, prostate-specific antigen and risk of prostate cancer. Nat Genet 2008; 40: 1032-4.

25 Eeles R, Giles G, Neal D, Muir K, Easton DF, for the PRACTICAL Consortium. Reply to 'Variation in KLK genes, prostate-specific antigen and risk of prostate cancer'. Nat Genet 2008; 40: 1035-6.

26 Verhaagh S, Schweifer N, Barlow DP, Zwart R. Cloning of the mouse and human solute carrier 22a3 (Slc22a3/SLC22A3) identifies a conserved cluster of three organic cation transporters on mouse chromosome 17 and human 6q26-q27. Genomics 1999; 55: 209-18.

27 Hidaka K, Caffrey JJ, Hua L, Zhang T, Falck JR, et al. An adjacent pair of human NUDT genes on chromosome $\mathrm{X}$ are preferentially expressed in testis and encode two new isoforms of diphosphoinositol polyphosphate phosphohydrolase. J Biol Chem 2002; 277: 32730-8.

28 Duggan D, Zheng SL, Knowlton M, Benitez D, Dimitrov L, et al. Two genome-wide association studies of aggressive prostate cancer implicate putative prostate tumor suppressor gene DAB2IP. J Natl Cancer Inst 2007; 99: 1836-44.

29 Sun J, Zheng SL, Wiklund F, Isaacs SD, Purcell LD, et al. Evidence for two independent prostate cancer risk-associated loci in the HNF1B gene at 17q12. Nat Genet 2008; 40: 1153-5. 